TITLE:

\title{
Vibrational energy relaxation of azulene in the S-2 state. I. Solvent species dependence
}

$\operatorname{AUTHOR}(S)$ :

Yamaguchi, T; Kimura, Y; Hirota, N

\section{CITATION:}

Yamaguchi, T ...[et al]. Vibrational energy relaxation of azulene in the S-2 state. I. Solvent species dependence. JOURNAL OF CHEMICAL PHYSICS 2000, 113(7): 2772-2783

\section{ISSUE DATE:}

2000-08-15

URL:

http://hdl.handle.net/2433/49897

\section{RIGHT:}

Copyright 2000 American Institute of Physics. This article may be downloaded for personal use only. Any other use requires prior permission of the author and the American Institute of Physics. 


\title{
Vibrational energy relaxation of azulene in the $S_{2}$ state. I. Solvent species dependence
}

\author{
T. Yamaguchi, ${ }^{\text {a) }}$ Y. Kimura, ${ }^{\text {b) }}$ and N. Hirota \\ Department of Chemistry, Graduate School of Science, Kyoto University, Kyoto 606-8502, Japan
}

(Received 24 January 2000; accepted 15 May 2000)

\begin{abstract}
We have measured the time-resolved fluorescence spectra of azulene in the $S_{2}$ state in compressed gases and in liquids. We have found that the band shape of the fluorescence changes significantly in the earlier time scale after the photoexcitation when large excess energy (about $6500 \mathrm{~cm}^{-1}$ ) is given. The change of the band shape is similar both in the compressed gases and in the liquids, although the time scales of the change are quite different. We have measured the excitation energy dependence of the fluorescence band shape of the isolated molecule separately, and shown that the time dependence of the fluorescence band shape in gases and liquids corresponds to the vibrational energy relaxation in the $S_{2}$ state. Comparing with the excitation energy dependence of the fluorescence band shape of the isolated molecule, we have succeeded in determining the transient vibrational excess energy. The vibrational energy relaxation rates in the $S_{2}$ state are 1-2 times faster than those in the ground state both in compressed gases and in liquids. (C) 2000 American Institute of Physics. [S0021-9606(00)50231-1]
\end{abstract}

\section{INTRODUCTION}

Vibrational energy transfer to the bath molecules is one of the fundamental reactions both in the gas phase ${ }^{1}$ and the liquid phase ${ }^{2,3}$ chemistry. A molecule can possess a large amount of intramolecular vibrational excess energy just after the photoexcitation or the chemical reaction. Since vibrationally "hot" molecules show different reactivity, we need to know the rate of the energy dissipation in order to understand chemical reactions. Therefore, many experimental and theoretical studies on the vibrational energy relaxation have been performed both in the gas phase and in solution.

In the gas phase, vibrational energy relaxation can be treated as a bimolecular reaction, and there have been two topics on the vibrational energy relaxation in the gas phase. One is the relationship between the relative relaxation rates and the molecular properties of both the solute and the bath molecules, ${ }^{4-13}$ and the other is the dependence of the efficiency of the vibrational energy transfer on the vibrational excess energy. ${ }^{4-7,14-17}$

Although the same problems are also important in the vibrational energy relaxation in solution, the situation appears more complicated in solution, since the vibrational energy relaxation may no longer be the bimolecular reaction due to the many-body effects in liquid. Many ideas on the vibrational energy relaxation in liquids have been proposed over the latter half of this century. The simplest idea among them is the isolated binary collision model (IBC model), where the vibrational energy relaxation in solution is treated as the bimolecular reaction between a solute and a solvent as in the gas phase reaction. ${ }^{2,3,5,18-29}$ This model has been used to analyze the experimental data since the earlier studies of the vibrational energy relaxation using the ultrasonic

\footnotetext{
${ }^{a)}$ Present address: Institute for Chemical Research, Kyoto University, Gokasho, Uji, Kyoto 611-0011, Japan.

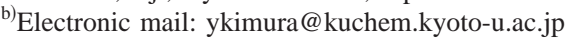

experiment. ${ }^{2,22,23}$ After the first proposal, some criticisms were given to the IBC model, ${ }^{2,30,31}$ and many models have been proposed which include the many-body nature of the liquid, for example, three-body interference, ${ }^{31}$ hydrodynamic model, ${ }^{32}$ and heat diffusion model. ${ }^{3,33,34,35(\mathrm{c})}$ In addition to these theoretical models, many numerical simulation studies have been performed in recent days owing to the great improvement of the performance and the availability of computers. ${ }^{19,27,29,36-39}$ However, we consider that even the validity of the simplest and the earliest model has not been made clear to date. It is required to examine the IBC model and clarify the relationship between the vibrational energy relaxation in the gas phase and in the liquid phase.

In a series of papers, we shall present our experimental results on the vibrational energy relaxation of azulene (inset of Fig. 1) in the $S_{2}$ state in gases, liquids, and so-called supercritical fluids. In paper I (this paper), we introduce our experimental method and present the results in gases and in liquids. In paper II ${ }^{40}$ we present the results in supercritical fluids and discuss the validity of the IBC model. Parts of the results of this work were already presented in the preceding letter ${ }^{41}$ although some of the results are modified.

Although the vibrational energy relaxation in the ground electronic state has been studied for various molecules, the number of the studies on the electronic excited state seems rather small compared with that in the ground state in the gas phase. The vibrational energy relaxation in the electronic excited state is more important in the solution chemistry. Since the vibrational energy relaxation in solution is very fast (within $100 \mathrm{ps)}$ ), only the fast photochemical reaction can compete with it, where the electronic excited states are likely to be involved. In addition, it is interesting to compare the vibrational energy relaxation in the excited states with that in the ground state in order to clarify the relationship between the relaxation rates and the properties of the solute molecules. For this purpose, it is desirable to measure the relaxation rates both in the ground and in the excited states of the 


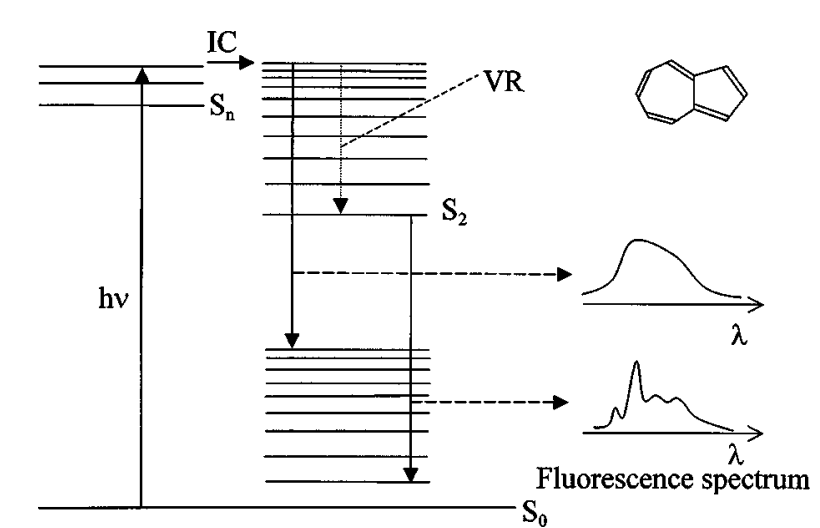

FIG. 1. Experimental scheme. IC, internal conversion; VR, vibrational energy relaxation. The structure of azulene is drawn at the right-upper side.

same molecules, because we can change the properties of the solute keeping the structure of the molecule almost the same. However, even in the gas phase, there are only a few molecules whose vibrational energy relaxation is measured both in the ground and the excited states. ${ }^{7,12,42}$ In solution, there is no case in which the relaxation from the vibrationally highly excited state is measured in both the ground and the electronic excited states to our best knowledge. An electronic excited state should relax to the ground state faster than the vibrational energy relaxation in order to create vibrationally "hot" ground states by internal conversion. On the other hand, the lifetime of an excited state should be longer than the vibrational energy relaxation in order to measure the vibrational energy relaxation in the excited state. These two conditions appear contradicting. However, azulene is a rare candidate to satisfy both by using two different electronic excited states so far as we know. The vibrational energy relaxation of azulene in the ground state is measured by many researchers both in gases ${ }^{4,5,6,43}$ and in liquids. ${ }^{3,5,20,33}$ Recently, Schwarzer et al. measured the vibrational energy relaxation of azulene in the ground state in supercritical ethane, carbon dioxide, and xenon. ${ }^{5}$ In this paper, these results are compared with our results on the vibrational energy relaxation in the $S_{2}$ state.

Azulene emits relatively strong and long-lived (about 2 ns) fluorescence from the $S_{2}$ state, contrary to the fact that the internal conversion from higher electronic excited states to the $S_{1}$ state is usually very fast and that the fluorescence from higher states is very weak (Kasha's Rule). The absorption and the fluorescence spectra of azulene are shown in Fig. 2. Due to the peculiar properties of the $S_{2}$ fluorescence of azulene, there are many spectroscopic and theoretical studies on it. ${ }^{44-47}$ For example, Hirata and Lim reported that the fluorescence band shape changes drastically with the excitation wavelength in the vapor phase. ${ }^{45}$ It means that the fluorescence band shape can be a measure for the intramolecular vibrational energy, and we can determine the time evolution of the intramolecular vibrational energy from the temporal change of the spectral band shapes. In this study, we measured the time-resolved fluorescence spectra of azulene in the $S_{2}$ state in compressed gases and in liquids. We found temporal changes of the spectrum bandshape both in compressed gases and solutions. We also measured the exci-
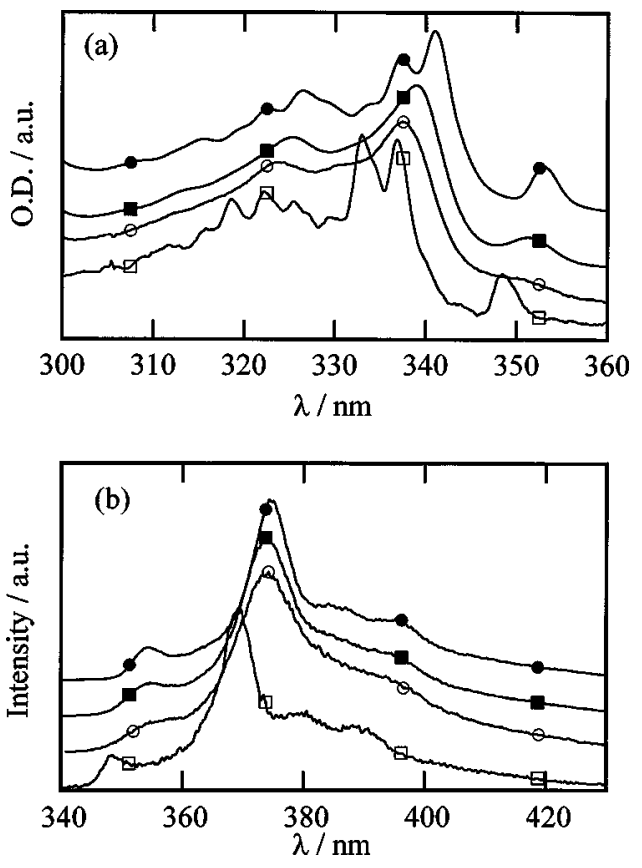

FIG. 2. $S_{0}-S_{2}$ electronic spectra of azulene. (a) Absorption spectra; (b) fluorescence spectra. From upper to lower, in cyclohexane, in methanol, in water, and in the vapor phase.

tation energy dependence of the fluorescence bandshape of an isolated molecule (vapor phase), and found that the time evolution of the spectrum band shape in solution corresponds to the loss of the intramolecular vibrational excess energy. By comparing the band shapes at different times with the "hot" spectra of an isolated molecule, we can determine the absolute value of the intramolecular vibrational excess energy. The experimental scheme is drawn in Fig. 1. Although it has been frequently reported that there is a temporal change of the fluorescence at $10 \mathrm{ps}$ scale after the photoexcitation in solution, which has been assigned to the vibrational energy relaxation of the excited state, ${ }^{3,48,49,50}$ this is the first study on the correspondence between the transient "hot" spectrum in solution and the "hot" fluorescence of the isolated molecule to our best knowledge. We shall note here that the vibrational energy relaxation rates of azulene in the $S_{2}$ state in rare gas matrices were already reported. ${ }^{51}$ Hereafter in this paper, we will show the studies on the vibrational energy relaxation of azulene in the $S_{2}$ state in compressed gases and in liquids. The results in supercritical fluids will be presented in Paper II. ${ }^{40}$

\section{EXPERIMENT}

The apparatus to measure the time-resolved fluorescence spectrum by a streak camera is described elsewhere. ${ }^{41,52}$ Briefly, the second harmonics of a mode-locked Nd:YAG laser (Coherent Antares 76) pumped a dye laser with a cavity dumper build in it (Coherent 700). Saturable absorber (DASBTI) was used to stabilize the dye laser when the fast relaxation in liquids was measured. ${ }^{53}$ The output pulse of the dye laser was about 7 ps width $(\sim 3$ ps width when saturable absorber was used), $\sim 8 \mathrm{~nJ}$, and $1.3 \mathrm{MHz}$ of repetition when operated at $569 \mathrm{~nm}$. After the polarization was adjusted by a 
half wave plate and a polarizer, the output of the dye laser was frequency doubled by a BBO crystal of $1 \mathrm{~mm}$ thickness. The obtained light of $285 \mathrm{~nm}$ was used for excitation. The polarization of the excitation light was adjusted to the magic angle so as to avoid the effect of rotational relaxation. ${ }^{54}$ The fluorescence was collected at the right angle and focused onto the slit of the spectrograph (Chromex 250IS). A quartz depolarizer was placed in front of the slit of the spectrograph and both of the polarizations were collected. The width of the slit was $100 \mu \mathrm{m}$ (optical slit width was about $3 \mathrm{~nm}$ ) in all the measurements. ${ }^{5}$ The spectra were obtained by a streak camera (Hamamatsu, C4334) operated at the photon counting mode. A small amount of the SHG of the Nd:YAG laser was separated to trigger the streak camera. The full-width at half-maximum (FWHM) of the response function was typically 30 ps when the streak camera was operated at the fastest sweep speed. We had to reduce the sweep speed in order to measure the slow vibrational energy relaxation in dilute gases, and the FWHM of the response function increases to $100 \mathrm{ps}$ in these experiments. The wavelength was calibrated by measuring the light from a low-pressure mercury lamp. ${ }^{55}$ The wavelength dependence of the sensitivity was corrected by measuring the reference sample (2-aminopyridine). ${ }^{56}$ The wavelength dependence of the time delay due to the mechanical artifact of the streak camera was corrected by using the time profile of the fluorescence of azulene in the vapor phase. The dye laser was operated at $569 \mathrm{~nm}$ in the measurement of the time-resolved spectra in solution. The wavelength of the output of the dye laser was swept from 566 to $676 \mathrm{~nm}$ in the measurement of the excitation wavelength dependence in the vapor phase. Rhodamine $6 \mathrm{G}$ was used at the wavelength shorter than $620 \mathrm{~nm}$, and DCM was used at the longer wavelength. Pyridine 1 was also used to operate at $674 \mathrm{~nm}$.

In order to measure the vibrational energy relaxation in the $S_{2}$ state, the relaxation rate should be faster than the lifetime of the $S_{2}$ state. Therefore we compressed buffer gases to increase the vibrational energy relaxation rates (0.4-10 MPa). The high-pressure optical cell used for the measurement in compressed gases is described elsewhere. ${ }^{57}$ The temperature of the cell was controlled at $341 \pm 1 \mathrm{~K}$ by flowing thermostated water through the cell and measured by a thermocouple immersed in the cell. The pressure of the cell was monitored by strain gages (Kyowa PGM $20 \mathrm{KH}$ for $<2$ $\mathrm{MPa}$, and Kyowa PGM $500 \mathrm{KH}$ for $>2 \mathrm{MPa}$ ), and the densities of the gases were calculated from the empirical equations of state. ${ }^{58}$ The concentration of azulene was $<10^{-4} \mathrm{~mol} \mathrm{dm}^{-3}$. The sample cell was purged twice by the sample gas before experiment. Helium, argon, xenon, nitrogen, ethane, and carbon dioxide were used as bath gases. We also measured the time-resolved fluorescence in oxygen in order to estimate the electronic quenching rate by oxygen. An ordinary $10 \mathrm{~mm}$ quartz cell was used in the measurement of liquid solutions. Cyclohexane, acetonitrile, methanol, ethylene glycol, and water were used as solvents. In measuring the fluorescence in the vapor phase of azulene, a small amount of azulene was enclosed in the quartz cell that was evacuated by a vacuum pump to $5 \times 10^{-3}$ Torr with azulene trapped by liquid nitrogen. Then the cell was immersed into
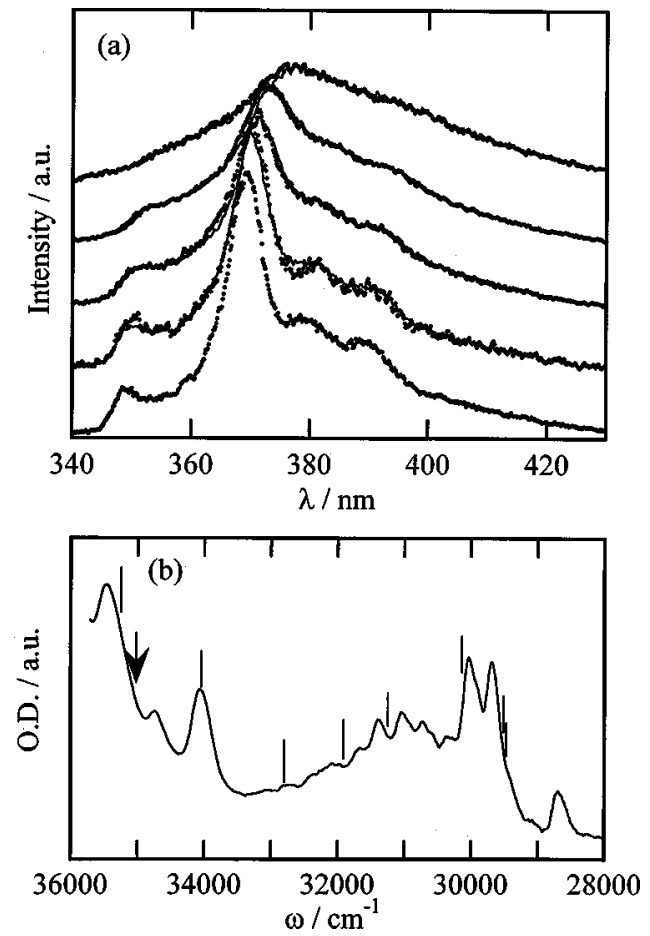

FIG. 3. (a) Excitation energy dependence of the fluorescence spectrum of azulene in the $S_{2}$ state. The excitation wavelengths are, from upper to lower, $283 \mathrm{~nm}, 305 \mathrm{~nm}, 320 \mathrm{~nm}$, and $338 \mathrm{~nm}$, respectively. The lowest one is the relaxed fluorescence in 20 bar of Ar. Filled circles, experiment; solid lines, fitted ones. (b) The absorption spectrum of azulene in the vapor phase. Vertical lines indicate the energy of the photon used to measure the excitation energy dependence of the fluorescence line shapes in the vapor phase. The arrow indicates the photon energy used in the experiments in solution.

an air bath that was temperature controlled at $343 \pm 1 \mathrm{~K}$. In measuring the fluorescence in water vapor, a small amount of water was also enclosed in the vacuum quartz cell together with a small amount of azulene. The cell was immersed into the air bath and the temperature was controlled at $343 \pm 1$ or $353 \pm 1 \mathrm{~K}$. The amount of the water was large enough that the pressure of water was calculated from the saturation vapor pressure. The decrease of the vapor pressure due to the solute was neglected because of the low solubility of azulene to water. The absorption spectra were measured by an UV absorption spectrometer (UV2500PC, Shimadzu).

Azulene (Nacalai Tesque) was purified twice by sublimation before use. Distilled water (Nacalai Tesque), cyclohexane, acetonitrile, methanol (Nacalai Tesque, spectroscopic grade), and ethylene glycol (Nacalai Tesque, guaranteed grade) were used as received. Helium (Iwatani, $>99.999 \%$ ), argon (Sumitomo Seika, >99.999\%), xenon (Iwatani, >99.995\%), nitrogen (Sumitomo Seika, $>99.999 \%$ ), ethane (Sumitomo Seika, >99\%), carbon dioxide (Sumitomo Seika, >99.98\%), and oxygen (Izumi, 99\%) were used without further purification.

\section{RESULTS}

\section{A. The excitation energy dependence of fluorescence in the vapor phase}

Figure 3(a) shows typical examples of the fluorescence bandshapes at different excitation wavelengths in the vapor 


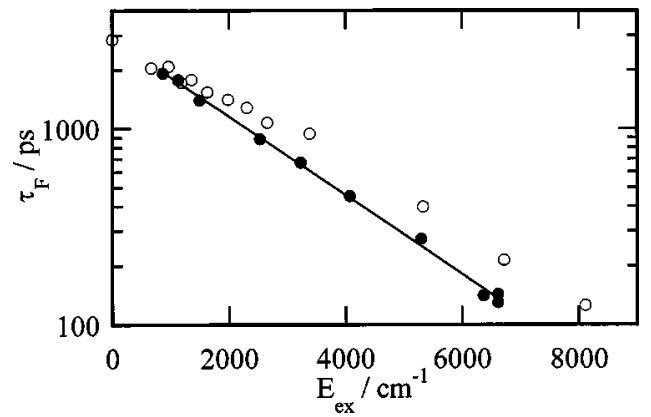

FIG. 4. Intramolecular vibrational excess energy dependence of the fluorescence lifetime in the vapor phase. $\bullet$, this work; $\bigcirc$, the lifetimes determined from the relative fluorescence quantum yield by Hirata and Lim (Ref. 45).

phase. We measured the fluorescence spectra at 283,285 , $294,305,313,320,331,337$, and $338 \mathrm{~nm}$. We did not detect meaningful time dependence of the fluorescence band shapes. The relaxed fluorescence in the dilute gas (argon, 20 bar) is also shown. The bandshapes of the relaxed fluorescence did not depend on buffer gases. The absorption spectrum of azulene is shown in Fig. 3(b), together with the photon energy used to measure the excitation energy dependence. As is clearly shown, the fluorescence spectrum is broad and structureless when excited at a shorter wavelength, i.e., the intramolecular vibrational excess energy is large. The fluorescence spectra become sharp and structured with a decrease of the intramolecular vibrational excess energy. In addition to the broadening, a small peak shift and the change of the relative intensity of the vibronic bands are observed. These changes are consistent with the result reported by Hirata and Lim. ${ }^{45}$

We also measured the excitation energy dependence of the fluorescence lifetime in the vapor phase. The results are shown in Fig. 4, together with the values determined by Hirata and Lim, from the relative fluorescence quantum yield. ${ }^{45}$ The fluorescence lifetime gets shorter with an increase of the intramolecular vibrational excess energy, and the excess energy dependence is almost exponential. Our excess energy dependence is a little larger compared with that of Hirata and Lim. ${ }^{45}$ We approximated the vibrational excess energy $\left(E_{\text {ex }}\right)$ dependence of the fluorescence lifetime $\left(\tau_{F}\right)$ by the following function,

$$
\tau_{F}=\tau_{F}^{0} \exp \left(-\alpha E_{\mathrm{ex}}\right)
$$

and obtained values are $\tau_{F}^{0}=2.9 \mathrm{~ns}$ and $\alpha=4.6 \times 10^{-4} \mathrm{~cm}$.

\section{B. Time-resolved fluorescence in gases and liquids}

Figure 5 shows the temporal change of the spectrum band shape of azulene $S_{2}$ fluorescence in compressed argon (20 bar) and methanol at the ambient condition. Although the time scales are quite different, the ways of the spectral change resemble to each other. Similar changes are found in all the compressed gases and liquids, although the time scale and the amount of the change depend on the species and the densities of the solvents. Compared with the excitation energy dependence of the fluorescence band shape in the vapor phase [Fig. 3(a)], it is noticed that the fluorescence band shapes at earlier times in solution have the characteristics of
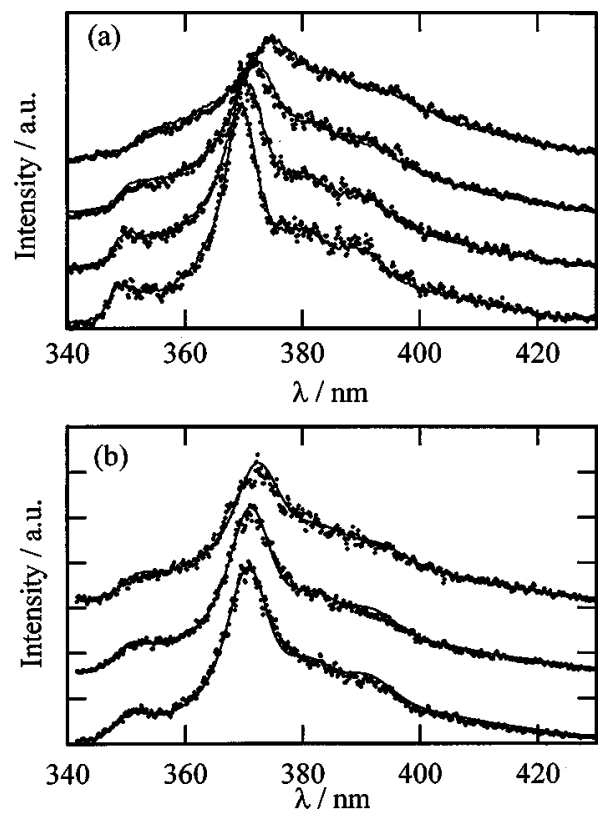

FIG. 5. Time-resolved fluorescence spectra of azulene. (a) In 20 bar of argon. From upper to lower, at 105 ps (estimated excess energy, 5320 $\left.\mathrm{cm}^{-1}\right), 306$ ps $\left(2890 \mathrm{~cm}^{-1}\right), 506$ ps $\left(1460 \mathrm{~cm}^{-1}\right)$, and $995 \mathrm{ps}\left(490 \mathrm{~cm}^{-1}\right)$. (b) In methanol. From upper to lower, at 0.4 ps $\left(2040 \mathrm{~cm}^{-1}\right), 21.2 \mathrm{ps}(520$ $\left.\mathrm{cm}^{-1}\right)$, and $41.9 \mathrm{ps}\left(40 \mathrm{~cm}^{-1}\right)$. Filled circles, observed; solid curves, fitted.

the vibrationally unrelaxed spectra, i.e., broad, structureless, small red shift, and the change of the relative intensity of the vibronic bands. Therefore we can assign the change of the fluorescence bandshape to the vibrational energy relaxation of azulene in the $S_{2}$ state.

In order to obtain the vibrational excess energy at each time delay, we compared the fluorescence line shapes with those obtained in vapor phase with different vibrational excess energy. In such a comparison, we have made a calibration curve to convert the fluorescence band shape to the intramolecular excess energy; we fitted the spectra observed in the vapor phase by an analytical function of the excess energy whose derivatives we can obtain easily. First, the fluorescence spectra were normalized by the integrated intensity. Next, the fluorescence intensity at each probe wavelength was fitted by a third order polynomial of the excess energy. ${ }^{59}$ In this procedure, we used the relaxed fluorescence in replace of the fluorescence excited at the $0-0$ band, and the fluorescence of zero excess energy was fixed. We used $348.2 \mathrm{~nm}$ for the wavelength of the $0-0$ band to calculate the intramolecular vibrational excess energy. The fitting curves are also shown in Fig. 3(a). The experimental spectra are reproduced quite well by the fitting curves.

By using this calibration function, we fitted the fluorescence spectrum at each delay time to determine the vibrational excess energy at each time. Since the relaxed fluorescence spectrum of azulene depends on solvents [Fig. 2(b)], we need to correct the spectrum change due to the solvent. In the correction, we took both the peak shift and the broadening into account. First, we simply shifted the reference spectra (spectra in the vapor phase). Next, we convoluted the reference spectra with the following Gaussian function:

$$
\exp \left(-\omega^{2} / 4 k_{B} T \lambda_{s}\right) / \sqrt{2 \pi k_{B} T \lambda_{s}},
$$



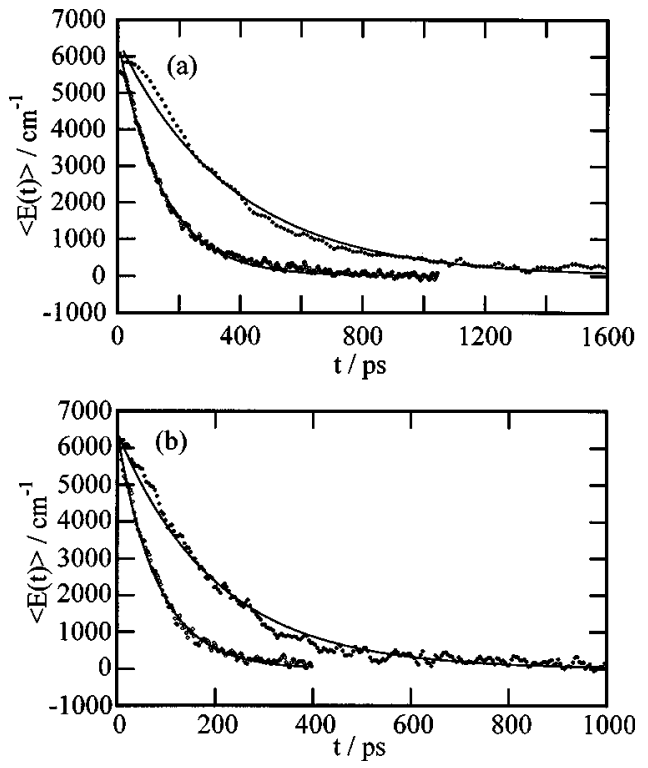

FIG. 6. Time dependence of the intramolecular vibrational excess energy in dilute gases. (a) Results in argon. Filled circles, experiment at 20 bar; open circles, that at 80 bar; solid curves, simulation. (b) Results in ethane. Filled circles, experiment at 8 bar; open circles, that at 21 bar; solid curves, simulation.

where $\omega, k_{B}, T$ and $\lambda_{s}$ stand for the frequency, the Boltzmann constant, the absolute temperature, and the solvent reorganization energy, respectively. After the correction, we fitted the transient spectra to the reference spectra by the least square method. The amounts of the shift and the broadening were determined so that the obtained vibrational excess energy reaches to zero after the completion of the vibrational energy relaxation. We assumed that the shift and the broadening do not depend on the vibrational excess energy. In compressed gases, no broadening was required and the amount of the shift was within $\pm 1 \mathrm{~nm}$. In the case of liquid solution, larger reorganization energies were required for polar and protic solvents. For example, $\lambda_{s}$ is about $70 \mathrm{~cm}^{-1}$ for methanol and about $150 \mathrm{~cm}^{-1}$ for water. The absolute values of the reorganization energies do not contradict with the solvent induced fluorescence Stokes shift (Fig. 2) qualitatively. The results of the fitting are shown in Fig. 5 (solid curves). The fitting procedure works well both in compressed gases and in liquid solutions.

\section{Vibrational energy relaxation in compressed gases}

The time dependence of the intramolecular vibrational energy obtained by this fitting procedure is shown in Fig. 6 . In the case of argon at 20 bar, the vibrational energy relaxation time is about 500 ps as seen in Fig. 6. Since we know the excitation wavelength $(285 \mathrm{~nm})$ and the wavelength of the $0-0$ band $(348.2 \mathrm{~nm})$, we can calculate the intramolecular vibrational excess energy just after the creation of the $S_{2}$ state $\left(6430 \mathrm{~cm}^{-1}\right)$. The initial vibrational energy obtained from the time-resolved spectrum agrees with the theoretical value well in the case of compressed gases. Therefore we consider that the vibrational energy dissipation does not occur in the time scale faster than the time resolution of our experiment in the case of compress gases. The time evolu-

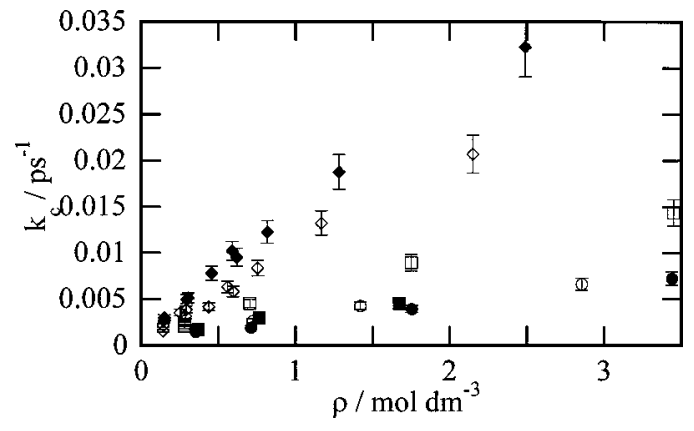

FIG. 7. Solvent density dependence of the vibrational energy relaxation rates $\left(k_{c}\right)$ in dilute gases. $\bullet$, helium; $\bigcirc$, argon; $\mathbf{\square}$, xenon; $\square$, nitrogen; $\bullet$, ethane; $\diamond$, carbon dioxide.

tion of the vibrational excess energy is reproduced by a single exponential function fairly well (solid curves in Fig. 6). The agreement is especially good for higher solvent densities. The deviations are found for lower solvent density around the vibrational excess energy of $5000 \mathrm{~cm}^{-1}$ and 1000 $\mathrm{cm}^{-1}$. The spectrum quality is worse at lower densities due to the lower solubility and the shorter excited-state lifetime. Since the vibrational excess energy dependence of the fluorescence band shape is relatively small around this excess energy, we consider this deviation is due to the fitting error of the fluorescence spectra. We interpret the time constant of this exponential function as the vibrational energy relaxation rates in compressed gases.

The vibrational energy relaxation rates determined by the band shape analysis are plotted against the solvent density in Fig. 7. The molecular gases work as efficient vibrational energy acceptors compared to rare gases. The comparison of the relaxation efficiency in the $S_{2}$ state with that in the $S_{0}$ state is given in Table I. Ideally, the biomolecular rates should be measured in the low-density limit. However, the relaxation rate is not proportional to the solvent density in this density region, and the measurement at the lower density is limited by the lifetime of the $S_{2}$ state unfortunately. Therefore, we compare the relaxation rates of $S_{0}$ and $S_{2}$ states at $1 \mathrm{~mol} \mathrm{dm}^{-3}$ instead. The rates in nitrogen are compared at $2 \mathrm{~mol} \mathrm{dm}^{-3}$ due to the lack of the data of the $S_{0}$ state. The relaxation rates of the $S_{0}$ state are drawn from Ref. 5. The Lennard-Jones collision frequency $\left(Z_{\mathrm{LJ}}\right)$ is calculated from the LJ parameters in Ref. 4(a), assuming the proportionality between the density and the collision frequency. ${ }^{60}$

TABLE I. The efficiency of the vibrational energy transfer per collision. The relaxation rates in the $S_{0}$ state are drawn from Ref. 5. The quenching rate at $1 \mathrm{~mol} \mathrm{dm}^{-3}$ is used except for water vapor (vapor pressure) and nitrogen $\left(2 \mathrm{~mol} \mathrm{dm}^{-3}\right)$. The collision frequency $\left(Z_{\mathrm{LJ}}\right)$ is obtained from the LJ parameter in Ref. 4(a).

\begin{tabular}{lccc}
\hline \hline Solvent gases & $k_{c}\left(S_{0}\right) / Z_{\mathrm{LJ}}\left(10^{-3}\right)$ & $k_{c}\left(S_{2}\right) / Z_{\mathrm{LJ}}\left(10^{-3}\right)$ & $k_{c}\left(S_{2}\right) / k_{c}\left(S_{0}\right)$ \\
\hline Helium & 4.9 & 4.7 & 1.0 \\
Argon & $\cdots$ & 10 & $\cdots$ \\
Xenon & 5.5 & 12 & 2.2 \\
Nitrogen & 6.9 & 14 & 2.0 \\
Ethane & 19 & 30 & 1.6 \\
Carbon dioxide & 13 & 28 & 2.2 \\
Water & $\cdots$ & 43 & $\cdots$ \\
\hline \hline
\end{tabular}




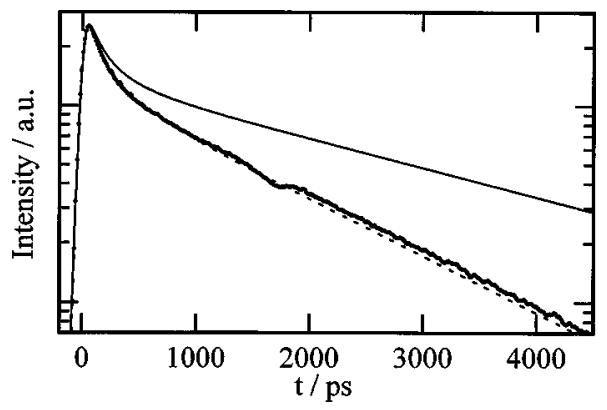

FIG. 8. Time dependence of the total fluorescence intensity in the 20 bar of argon. Filled circles, experiment; solid curve, simulated by the excess energy dependence of the fluorescence lifetime in the vapor phase; broken curve, fitted by adjusting parameters (see text).

Before the presentation of the results in liquids, we will discuss the time profile of the fluorescence intensity. In Fig. 8 , we show the time dependence of the total fluorescence intensity in argon. The longitudinal axis is drawn in the log scale. A small dip near $1800 \mathrm{ps}$ is an artifact due to the damage of the MCP plate of our streak camera. As is clearly seen, the fluorescence intensity does not decay with a single time constant, but the decay rate becomes slower at later times. It is consistent with the fact that the $S_{2}$ lifetime becomes shorter with an increase of the intramolecular vibrational excess energy (Fig. 4). The fluorescence decays faster at earlier times because a large amount of the vibrational excess energy is stored in the solute molecule. The nonexponential decay is found in all the gases, and the amplitudes of the fast components become smaller with an increase of the solvent density, which is consistent with the above idea. ${ }^{7,61}$

In principle, we can determine the vibrational energy decay rate by the analysis of the fluorescence intensity, as is demonstrated for the vibrational energy relaxation rates of $T_{1}$ pyrazine by McDowell et al., although in their case they used the excess energy dependence of the intersystem crossing rate. ${ }^{7}$ Here, we tried to simulate the time dependence of the total fluorescence intensity. In the simulation, we first assume exponential dependence of the fluorescence lifetime on the intramolecular vibrational energy [Eq. (1)]. Therefore, the time evolution of the fluorescence intensity $(f(t))$ obeys the following equation:

$$
\frac{d}{d t} f(t)=-f(t) / \tau_{F}(e(t)),
$$

where $e(t)$ stands for the excess energy possessed by the excited molecules, and $\tau_{F}$ is described by Eq. (1). The time evolution of $e(t)$ is assumed to be single exponential as follows:

$$
\frac{d}{d t} e(t)=-k_{c} e(t)
$$

where $k_{c}$ is the vibrational energy relaxation rate. In the simulation, we use $k_{c}$ determined by the band shape analysis, and $e(0)$ is fixed to $6430 \mathrm{~cm}^{-1}$. The response function is assumed to be the following form:

$$
g(t) \propto \operatorname{sech}^{2}\left(\frac{t-t_{0}}{\tau_{R}}\right),
$$
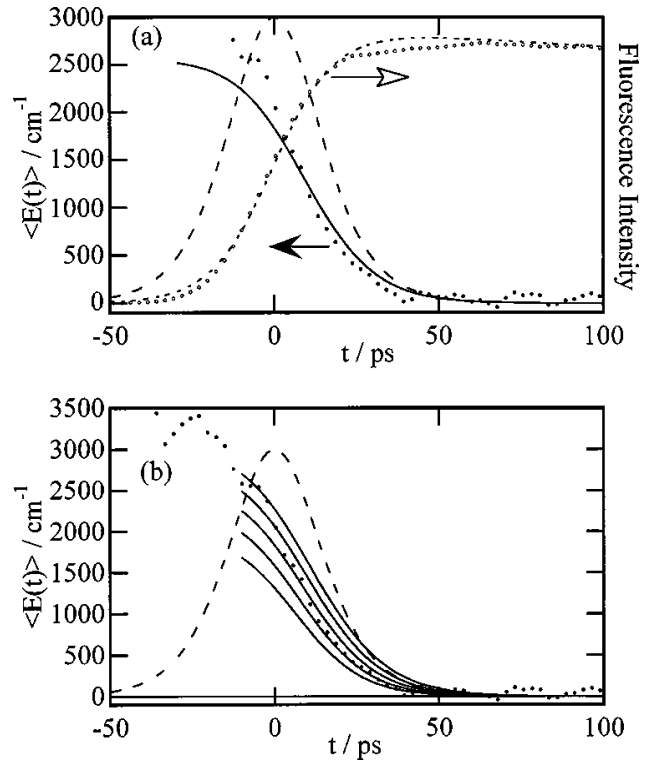

FIG. 9. Time dependence of the intramolecular vibrational excess energy in methanol. (a) Filled circles, experimental excess energy; solid curve, simulated excess energy, open circles, experimental fluorescence intensity; dotted lines, simulated fluorescence intensity; broken line, response function. (b) Simulation of $\langle E(t)\rangle$ with various relaxation time. Solid curves are the simulated ones. The relaxation times are, from upper to lower, $8 \mathrm{ps}, 7 \mathrm{ps}, 6$ ps, $5 \mathrm{ps}$, and $4 \mathrm{ps}$, respectively. Filled circles, experiment; broken line, response function.

where $t_{0}$ is the peak of the response function, and $\tau_{R}$ is a measure of the time resolution. The experimentally observed total fluorescence intensity $\left(I_{F}(t)\right)$ is expected to be described by the following equation:

$$
I_{F}(t)=\int_{-\infty}^{t} d t^{\prime} g\left(t^{\prime}\right) f\left(t-t^{\prime}\right) .
$$

At first, we tried to reproduce $I_{F}(t)$ with the vibrational excess energy dependence obtained from the experiment in the vapor phase $\left(\alpha=4.6 \times 10^{-4} \mathrm{~cm}\right.$ and $\left.\tau_{F}^{0}=2.9 \mathrm{~ns}\right)$. However, the simulation does not work at all (the solid curve in Fig. 8). Even the fluorescence lifetime after the vibrational relaxation (1.5 ns in 20 bar of argon) disagrees. $I_{F}(t)$ could be simulated only by empirically adjusting $\alpha$ and $\tau_{F}^{0}$ only to evaluate the response function, and the results are shown in Fig. 8 ( $\alpha=3.2 \times 10^{-4} \mathrm{~cm}$ and $\left.\tau_{F}^{0}=1.5 \mathrm{~ns}\right)$. The $I_{F}(t)$ 's for different densities and different gases are also reproduced by adjusting $\alpha=3.2-4.6 \times 10^{-4} \mathrm{~cm}$ and optimizing $\tau_{F}^{0}$. Therefore, we utilized the fluorescence intensity only to estimate the system response function. The origin of the discrepancy will be discussed in Sec. IV A.

\section{Vibrational energy relaxation in solution}

We show in Fig. 9(a) the time profile of the intramolecular vibrational energy of azulene in the $S_{2}$ state in methanol determined from the spectrum band shape (filled circles). The vibrational energy relaxation is almost completed within the instrumental response function. Therefore we cannot determine the relaxation rates simply by fitting the time profile of the excess energy to an exponential function, as is done in the case of compressed gases. Hence we made a convolution 
TABLE II. The vibrational energy relaxation times in liquids. The estimated errors are about $\pm 1 \mathrm{ps}$ in the case of the $S_{2}$ state.

\begin{tabular}{lccc}
\hline \hline \multicolumn{1}{c}{ Solvents } & $k_{c}\left(S_{0}\right)^{-1} / \mathrm{ps}^{\mathrm{a}}$ & $k_{c}\left(S_{2}\right)^{-1 / \mathrm{ps}}$ & $k_{c}\left(S_{2}\right) / k_{c}\left(S_{0}\right)$ \\
\hline Cyclohexane & 13.7 & 11 & 1.3 \\
Acetonitrile & 14.6 & 12 & 1.3 \\
Methanol & 8.3 & 5.8 & 1.4 \\
Ethylene glycol & 7.2 & 3.5 & 2.1 \\
Water & $\cdots$ & 2.3 & $\cdots$ \\
\hline \hline
\end{tabular}

${ }^{\mathrm{a}}$ Reference 5 .

with the system response function as described below. First, we assumed that the vibrational excess energy obtained from the spectrum band shape corresponds to the average vibrational excess energy possessed by the excited molecules $(\langle E(t)\rangle)$. Then $\langle E(t)\rangle$ is expected be described by the following equation:

$$
I_{F}(t)\langle E(t)\rangle=\int_{-\infty}^{t} d t^{\prime} g\left(t^{\prime}\right) f\left(t-t^{\prime}\right) e\left(t-t^{\prime}\right),
$$

where $I_{F}(t), g(t), f(t)$, and $e(t)$ are defined in the previous subsection. $I_{F}(t)$ is described by Eq. (6). From Eqs. (6) and (7), we obtained the relaxation rate $k_{c}$. Equation (7) is not exact in the presence of the excess energy dependence of the excited state lifetime. However, as will be shown in Appendix, this equation holds quantitatively well in our experimental condition. In the actual fitting procedure, we guess $k_{c}$ at first, and optimize $I_{F}(t)$ by Eqs. (3)-(6) in order to obtain $t_{0}, \tau_{R}$, and $\tau_{F}^{0}$. Next, we obtain $k_{c}$ from $I_{F}(t)\langle E(t)\rangle$ by Eqs. (3), (4), and (7). Then, above two fitting procedures are repeated until the self-consistent values are obtained. The value of $\alpha$ was fixed to $4.6 \times 10^{-4} \mathrm{~cm}$. The change of $\alpha$ did not lead to a meaningful change of the relaxation rate, because the internal conversion from the vibrationally "hot" state is more than ten times slower than vibrational energy relaxation in liquid. The initial value of the vibrational excess energy was fixed to the same value as in compressed gases, $6430 \mathrm{~cm}^{-1}$, neglecting the spectral shift in solution [about $+5 \mathrm{~nm}\left(\sim-400 \mathrm{~cm}^{-1}\right)$ for fluorescence]. The fitting curves and the response function are shown in Fig. 9(a). Both $I_{F}(t)$ and $\langle E(t)\rangle$ are reproduced fairly well by this analysis. In Fig. 9(b), we simulated $\langle E(t)\rangle$ with different values of $k_{c}$. The fitting error is estimated to be about \pm 1 ps. To be noted is that we could determine the relaxation rate because we evaluated the absolute values of the intramolecular vibrational excess energy, and assumed the single exponential decay function. Any one of the five curves in Fig. 9(b) can reproduce the experiment fairly well if the absolute values of the excess energy are scaled. The relaxation rates in the liquids are summarized in Table II.

\section{E. Vibrational energy relaxation in water vapor}

As is shown in Table II, the vibrational energy relaxation becomes faster in protic solvents. The fast vibrational cooling in hydrogen bonding liquids is also found in other systems. ${ }^{35,37,38,62,63}$ On the other hand, it is widely known that the water molecule acts as an efficient vibrational quencher in the gas phase. ${ }^{7,8}$ Therefore, we tried to measure
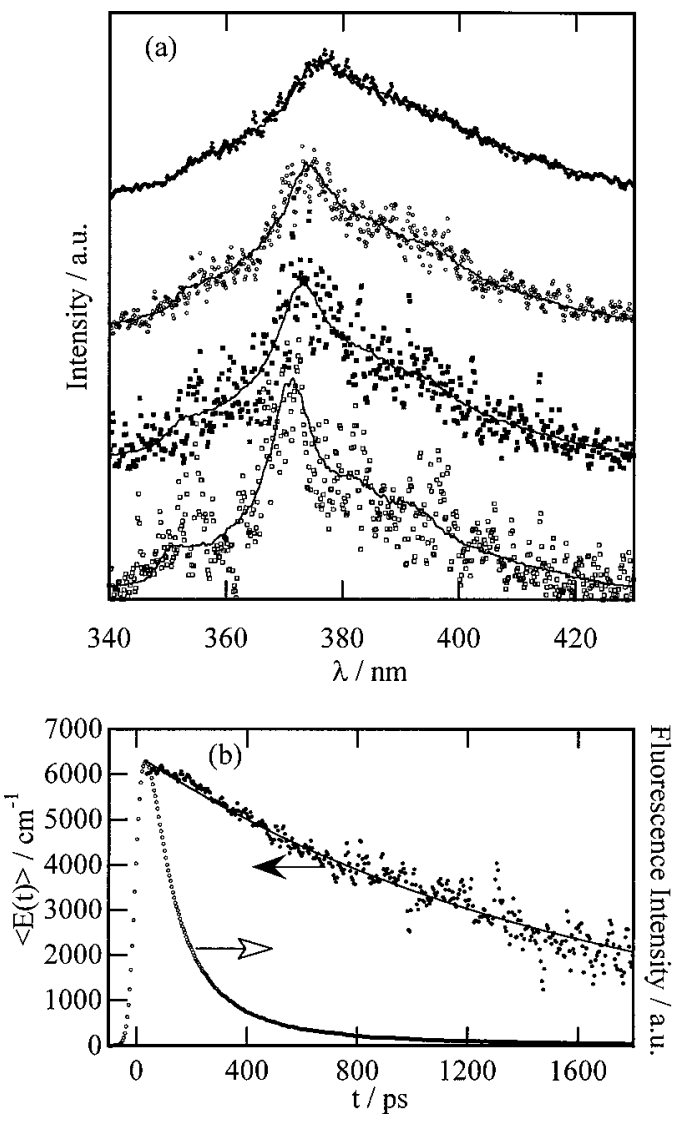

FIG. 10. Vibrational energy relaxation in the saturated water vapor at 353 K. (a) Time-resolved spectra. From upper to lower, at $97 \mathrm{ps}$ (estimated excess energy, $\left.6070 \mathrm{~cm}^{-1}\right), 499 \mathrm{ps}\left(4560 \mathrm{~cm}^{-1}\right), 1004 \mathrm{ps}\left(3810 \mathrm{~cm}^{-1}\right)$, and $1504 \mathrm{ps}\left(2250 \mathrm{~cm}^{-1}\right)$, respectively. Marks, experiment; solid curves, fitting. (b) Time dependence of the vibrational excess energy. Filled circles, experiment, solid curve, fitting; broken curve, fluorescence intensity.

the vibrational energy relaxation of azulene in the $S_{2}$ state in water vapor in order to understand the reason for the fast energy relaxation in liquid water.

The time-resolved spectra in the saturated water vapor at $353 \mathrm{~K}$ are shown in Fig. 10(a). The time profiles of the fluorescence intensity and the intramolecular vibrational excess energies are shown in Fig. 10(b). Due to the lower pressure of water vapor at $353 \mathrm{~K}$ ( 355 Torr), the vibrational energy relaxation is slower than those in other compressed gases in this study. As a result, the population of the $S_{2}$ state disappears before the vibrational energy relaxation completes, which makes the spectra at $t>1$ ns noisy due to the small intensity. However, we consider that we can estimate the relaxation rate roughly from this experiment, and we approximate $\langle E(t)\rangle$ to an exponential function [solid curve in Fig. 10(b)]. The value of $\langle E(t)\rangle$ is fixed to $6430 \mathrm{~cm}^{-1}$. The vibrational energy relaxation time is estimated to be $1.6 \mathrm{~ns}$. We performed the same experiment at $343 \mathrm{~K}$, where the saturated vapor pressure is 234 Torr, and the vibrational energy relaxation time $2.0 \mathrm{~ns}$. By averaging these data, we calculate the vibrational quenching efficiency per collision, which is shown in Table I. Water is an efficient vibrational quencher also for azulene in the $S_{2}$ state. 


\section{DISCUSSION}

\section{A. The effect of other relaxation processes in the excited states}

In the analysis in Sec. III, we have implicitly made some assumptions on the relaxation processes in the excited state. First, the internal conversion from the higher singlet excited states to the $S_{2}$ state is assumed to be very fast. The internal conversion from higher electronic excited states is generally very fast ( $S_{2}$ azulene is a famous exception), and the fluorescence from the $S_{3}$ or the $S_{4}$ state was not detected both in the gas and the liquid phases. ${ }^{44}$ Therefore, we believe that the internal conversion to the $S_{2}$ state is completed within sub-ps.

The next assumption is the fast intramolecular vibrational redistribution (IVR) in the $S_{2}$ state. We have evaluated the transient vibrational excess energy from a comparison between the transient spectra and the time-integrated hot spectra in the vapor phase. However, since the fluorescence spectra are different before and after IVR, ${ }^{48}$ the transient spectra of the solute molecule is not characterized only by the intramolecular vibrational excess energy. It is usually assumed in the vibrational cooling experiment that IVR completes within sub-ps in solution. Ohta et al. measured the time-resolved fluorescence spectrum of Coumarin 481 by fluorescence up-conversion method, and found that IVR is already completed just after the internal conversion from the higher electronic excited state. ${ }^{48}$ Sarkar et al. found that the anisotropy of the fluorescence of tetracene in the $S_{1}$ state decays within $1 \mathrm{ps}$, which they ascribed to IVR in the $S_{1}$ state. ${ }^{49}$ On the other hand, nonstatistical (IVR incomplete) vibrational population is sometimes observed by the timeresolved resonance Raman spectroscopy. ${ }^{64,65}$ However, fast IVR in the gas phase is reported in the case of azulene. In the jet-cooled experiment, Demmar et al. found that IVR of azulene in the $S_{2}$ state is faster than $30 \mathrm{ps}$ with a vibrational excess energy higher than $1950 \mathrm{~cm}^{-1},{ }^{66}$ and Diau et al. reported that the rate of IVR of azulene in the $S_{2}$ state is $350 \mathrm{fs}$ with an excess energy of $3800 \mathrm{~cm}^{-1} \cdot{ }^{67}$ In our experiment, we could not find any significant change of the fluorescence spectra of the isolated azulene within the signal to noise ratio of our experiment. Therefore, we consider that IVR in the $S_{2}$ state is fast enough that the state of the solute molecule is characterized well solely by the intramolecular vibrational excess energy at the vibrationally highly excited state. IVR may cause some errors in the estimation of $\langle E(t)\rangle$ at small values.

The third assumption is the neglect of the solvation dynamics in polar solution. Although the solvent shift of the $S_{2}$ spectra of azulene is small, the fluorescence Stokes shift is detectable in polar solvents such as water $(\sim 3 \mathrm{~nm})$. Therefore, solvation dynamics such as dynamic Stokes shift may affect the observed transient spectra in polar solvents, which we did not take into account in the analysis of the transient spectra. However, the temporal changes of the fluorescence bandshape observed in polar solvents are not explained solely by the solvation dynamics. The solvation dynamics should appear as a red shift due to the energy dissipation. It should also appear as the broadening, not the narrowing, of the fluorescence spectrum, since a narrow band laser selects particular solvation states. However, what we observed is a blue shift and a narrowing. In addition, the changes in the relative intensities of the vibronic bands are not likely explained by the solvation dynamics. Therefore, the temporal change of the fluorescence bandshape shown in Fig. 5(b) is dominated by the vibrational energy relaxation. However, there may be some contributions from the solvation dynamics in slowly relaxing solvents such as methanol or ethylene glycol. ${ }^{68}$ The effect of solvation dynamics will act to decrease the apparent excess energy and to increase the apparent relaxation rates. However, we neglect the effect of solvation dynamics simply because it is not distinguishable experimentally from the vibrational cooling.

In connection with the solvation dynamics, solventinduced thermochromism should be discussed as a candidate that can change the fluorescence band shape. The increase of the local solvent temperature is expected after the heat release to the solvent. The solvation structure under the elevated temperature is different from that of the initial temperature, which can alter the fluorescence band shape. For example, Eq. (2) predicts an increase of the fluorescence bandwidth. However, we consider that this effect is small in most cases of our study, since the absolute value of the solvent reorganization energy itself is small. The effect of the solvent-induced thermochromism may be large in highly polar solvents as water. However, we did not take this effect into account, because it is difficult to separate experimentally.

Finally, we should comment on the disagreement of the fluorescence lifetimes in the buffer gases and in the isolated state (Fig. 8). We suspected that a small amount of oxygen that remained in the cell quenched the $S_{2}$ state of azulene. In order to test this idea, we measured the fluorescence lifetime in compressed oxygen (1-4 bar) and determined the bimolecular quenching rate. The rapid decrease of the lifetime was found with an increase of the pressure of oxygen, and the lifetime becomes 30 ps at 4 bar of oxygen. The fluorescence band shape was the typical one for the vibrationally unrelaxed molecule and did not change with time. Therefore, the electronic quenching occurs much faster than the vibrational quenching in compressed oxygen. The obtained quenching rate of the $S_{2}$ state is $5.6 \mathrm{~ns}^{-1} \mathrm{bar}^{-1}$, which means that the quenching probability per collision is 0.5 if we calculate the collision frequency from the $\mathrm{LJ}$ parameter listed in Ref. 4(a). This value is close to the value reported for the $S_{1}$ authracence (0.3). ${ }^{69}$ Although the quenching rate may depend on the intramolecular vibrational energy, we use the above rate as a measure of the rate for the vibrationally cool molecule. If we ascribe the discrepancy of the relaxed fluorescence lifetime in Ar to that of vapor solely to the contamination of the oxygen, the partial pressure of oxygen is estimated to be 0.05 bar. Although we consider that the 0.05 bar of oxygen is too large, the contamination of oxygen can be one of the reasons of the discrepancy of the relaxed lifetime. 
In addition, the solvent effects on the lifetime may exist since our experimental condition is not so dilute.

\section{B. The time profile of the vibrational energy relaxation}

The experimental results of $\langle E(t)\rangle$ in compressed gases (Fig. 6) are almost reproduced by a single exponential function in Eq. (4), although there are small deviations. In the gas phase, the functional form of $\langle E(t)\rangle$ is related to the excess energy $(\langle E\rangle)$ dependence of the energy transfer efficiency per collision $(\langle\Delta E\rangle)$, which is one of the major topics in the gas phase chemistry. The single exponential form of $\langle E(t)\rangle$ means that $\langle\Delta E\rangle$ is proportional to $\langle E\rangle$. The excess energy dependence of $\langle\Delta E\rangle$ has been measured for many molecules, and many polyatomic molecules show the proportionality between $\langle E\rangle$ and $\langle\Delta E\rangle{ }^{5,6}$ Although thresholds are found for some molecules, they belong to rather special cases, since they are explained in terms of the coupling with upper electronic states. ${ }^{7,15,17}$

Since so many molecules show the proportionality between $\langle E\rangle$ and $\langle\Delta E\rangle$, there must be a simple logic underlying. Here we show an explanation by the simplest model of the vibrational energy relaxation. Suppose that a harmonic oscillator is immersed in the heat bath (solvent molecules). The perturbation from the heat bath on the oscillator is linear with respect to the coordinate of the harmonic oscillator [i.e., the form of $F(t) x$, where $x$ is the coordinate of the oscillator, and $F(t)$ is called force]. In this model, the vibrational energy relaxation by only one phonon is allowed, and the downward transition probability is proportional to the vibrational quantum number. It means the proportionality between $\langle E\rangle$ and $\langle\Delta E\rangle$, since the number of phonon is proportional to the vibrational energy. The above discussion holds irrespective of the statistical properties of $F(t) .^{70}$

We approximated $\langle E(t)\rangle$ as an exponential function also in liquids. It is nothing more than an approximation in this case, since we cannot determine the functional form of $\langle E(t)\rangle$ in liquids due to the low time resolution of our apparatus. The vibrational energy relaxation in liquids is sometimes proposed to be multistep processes, ${ }^{3,33-35,64}$ although no one observed the multi-step energy relaxation directly so far as we know. Schwarzer et al. measured the vibrational energy relaxation of $S_{0}$ azulene in supercritical fluids, and reported that the decay of the hot band is a single exponential at any density from gaslike to liquidlike ones. ${ }^{5}$ They reported the single exponential dependence also in liquids. For the sake of the comparison with their results, we employed the single exponential function as $\langle E(t)\rangle$ in liquids.

\section{The solute and the solvent dependence of the energy relaxation rates in compressed gases}

In this work, we found that the vibrational energy quenching of azulene in the $S_{2}$ state is 1-2 times as efficient as that of azulene in the ground state in compressed gases. The fast energy relaxation in the electronic excited state has already been found in the case of $T_{1}$ pyrazine. Weisman et al. measured the vibrational energy relaxation of $T_{1}$ pyrazine by the competitive radiationless decay method, and found that the vibrational energy relaxation of $T_{1}$ pyrazine is about seven times faster than that of $S_{0}$ pyrazine in the same gas. $^{7}$ They proposed three reasons for the faster vibrational energy relaxation in the $T_{1}$ state. The first one is the decrease of the intramolecular vibrational frequency. Since the characteristic frequency of the classical solvent motion is much lower than the frequency of the intramolecular vibration, the lower frequency mode is likely to relax faster. The second one is the increase of the anharmonicity of the intramolecular vibration in the electronic excitation. The last one is the enhancement of the coupling matrix elements due to the vibronic coupling to the $T_{2}$ state, which they proposed is the major reason for the fast relaxation of $T_{1}$ pyrazine, because they found a threshold near the energy level of the $T_{2}$ state. We consider that the first two reasons apply also to the case of azulene. Fujii et al. reported the decrease of the intramolecular vibrational frequency in the $S_{2}$ state. ${ }^{44}$ For example, $\nu_{39}$ shifts from $326 \mathrm{~cm}^{-1}$ to $236 \mathrm{~cm}^{-1}$, and $\nu_{17}$ does from $402 \mathrm{~cm}^{-1}$ to $372 \mathrm{~cm}^{-1}$. Although there is a threshold near the $T_{2}$ level in the case of $T_{1}$ pyrazine, we could not find any trace of the threshold near the $S_{3}$ level in the case of $S_{2}$ azulene, and what we mainly observed is the vibrational energy relaxation below the $S_{3}$ level. Therefore, the third reason for $T_{1}$ pyrazine by Weisman et al. does not apply to the vibrational energy relaxation of the present case. This may be the reason for the different degree of the enhancement of vibrational energy relaxation in the electronic excited state.

The solvent dependence of the quenching efficiency is another problem. Roughly speaking, the tendency among the solvent species is similar to those for other solute molecules. The molecular gases act as efficient quenchers compared with rare gases. The quenching efficiency of helium is lower than that of argon and xenon. Water acts as a very efficient quencher. However, when closely examined, the quenching efficiency does not appear to be determined solely by the properties of the solvent molecules, since the ratio of the quenching efficiencies in the $S_{0}$ and $S_{2}$ states varies with solvent species. We do not have any further explanation of this difference at present. More experimental and theoretical studies will be required to clarify the meanings of this difference.

We would like to comment here on the importance of $V-V$ transfer in the case of molecular quenchers. Jalenak et al. measured the vibrational energy relaxation of $S_{0}$ azulene in gaseous carbon dioxide by the time-resolved highresolution IR spectroscopy. ${ }^{43}$ In their experiment, the vibrational hot bands, the rotational branches, and the Doppler broadening of the solvent molecules were measured and they could determine the relative importance of the accepting modes of solvents. They estimated that the ratio of the $V-V$ transfer to the total energy transfer is about $25 \%$. Although the large vibrational quenching efficiency of molecular gases is sometimes attributed to the existence of the intramolecular vibrational modes, their result indicates that the $V-V$ transfer alone cannot explain the high quenching efficiency of carbon dioxide to azulene compared with those of rare gas molecules. However, Fraelich et al. studied the vibrational energy relaxation of $S_{0}$ pyrazine in water vapor by a similar method, and concluded that the large quenching efficiency of water is due to the channels other than the rotational and the 
translational modes. ${ }^{8}$ Anyway, we hope that this kind of experiments will clarify the mechanism of the vibrational energy relaxation by the molecular gases.

\section{The fast vibrational energy relaxation in the hydrogen bonding liquids}

It is clearly seen from Table II that the vibrational energy relaxation of azulene in the $S_{2}$ state is enhanced in the protic solvents. The fast vibrational energy relaxation in hydrogen bonding solvents, especially in liquid water, is found for many other systems, and they are usually attributed to the hydrogen bonding. However, it is somewhat ambiguous whether the solute-solvent or the solvent-solvent interaction (hydrogen bonding) is important. It is widely known that the correlation between water molecules due to the hydrogen bonding are strong enough that they have large influence on the solvation of the solute molecule, for example, the positive and the negative hydration, or the hydrophobic hydration. Therefore, the hydrogen bonding between water molecules can also affect the vibrational energy relaxation of a solute in liquid water. On the other hand, the vibrational quenching efficiency of a water molecule per collision is large in the gas phase. In addition, the solvent number density of liquid water is larger than those of organic solvents. Therefore, the above two factors alone may explain the fast vibrational energy relaxation in liquid water.

In order to clarify the effect of the hydrogen bonding between solvents, we should estimate the relaxation rate in the absence of the hydrogen bonding between the water molecules. For this purpose, we tried to compare the vibrational energy relaxation in liquid water and in compressed helium. Since the LJ radius of water $(0.271 \mathrm{~nm})$ is similar to that of helium $(0.255 \mathrm{~nm}),{ }^{4(\mathrm{a})}$ we employed a rough approximation that the collision frequency between azulene and helium differs from that between azulene and water only by the difference of the reduced mass. Unfortunately, we do not have the vibrational energy relaxation rate of azulene in helium at the same density as the liquid water $\left(55 \mathrm{~mol} \mathrm{dm}^{-3}\right)$. If we extrapolate the linear density dependence of the vibrational cooling rates of $S_{0}$ azulene in compressed helium in Ref. 5, the vibrational cooling time at $55 \mathrm{~mol} \mathrm{dm}{ }^{-3}$ is estimated to be 9 ps. Using this value, the IBC estimation of the cooling rate in liquid water is about $2 \mathrm{ps}$, which happens to agree with the experiment. This indicates the importance of the gas phase quenching efficiency in the interpretation of the fast vibrational cooling in liquid water, although it does not necessarily exclude the importance of the hydrogen bonding between solvent molecules, considering the rough estimation and the experimental uncertainty.

\section{SUMMARY}

In this work, we measured the time-resolved fluorescence spectra of azulene in the $S_{2}$ state in compressed gases and liquids. The band shapes of the spectra change with time at the earlier time after the photoexcitation both in gases and liquids, although the time scale of the change is quite different. From the comparison with the excitation energy dependence of the fluorescence band shape of the isolated mol- ecule, we assigned the temporal evolution of the fluorescence band shape to the vibrational energy relaxation. By analyzing the temporal change of the fluorescence band shape, we determined the vibrational energy relaxation rates in the $S_{2}$ state, which is 1-2 times as large as those in the $S_{0}$ state. We proposed that the low frequency shift of the intramolecular vibrational mode on the electronic excitation is a candidate for the reason of the fast vibrational energy relaxation in the $S_{2}$ state. In the liquid phase, we found that the vibrational energy relaxation in the $S_{2}$ state is enhanced in the hydrogen bonding solvents. We also found that the water molecule is an efficient vibrational quencher for azulene in the $S_{2}$ state even in the vapor phase. We showed that the large quenching efficiency in the gas phase might be a reason for the fast energy relaxation in liquid water. In order to clarify the relationship between the vibrational energy relaxation in the gas phase and that in the liquid phase, we will present the study on the vibrational energy relaxation in the $S_{2}$ state of azulene in so-called supercritical fluids in the successive Paper (II). ${ }^{40}$

\section{ACKNOWLEDGMENTS}

We are grateful to Dr. Y. Yoshimura (Kyoto University) for the use of the UV absorption spectrometer. T. Y. gratefully acknowledges a research fellowship from the Japan Society for the Promotion of Science (JSPS) for Young Scientists. This work is supported by CREST (Core Research for Evolutional Science and Technology) of Japan Science and Technology (JST) and by the Research Grant-in-Aid from the Ministry of Education, Science and Culture (No. 11640504).

\section{APPENDIX}

As was mentioned in the text, Eq. (7) is not an exact relationship, but a mere approximation under the presence of the excess energy dependent fluorescence lifetime. Suppose that there is a distribution of the vibrational excess energy in an ensemble of the excited molecules. Since the higher energy part of the distribution decays faster due to the smaller lifetime, the averaged excess energy decreases with time even in the presence of no buffer gas. Therefore, the excess energy dependence of the fluorescence lifetime makes the apparent relaxation rate faster. This effect becomes larger when the vibrational energy relaxation is slow, since the electronic relaxation can compete with the vibrational energy relaxation in such a case. In order to estimate this effect quantitatively, we have made a Monte Carlo simulation.

In this simulation, a solute molecule is subject to uncorrelated collisions with buffer gas molecules. The distribution of the energy loss $(P(E, \Delta E))$ at a collision is assumed to be exponential as follows:

$P(E, \Delta E)= \begin{cases}\exp (-\Delta E /\langle\Delta E\rangle(E)) /\langle\Delta E\rangle(E) \quad(\Delta E>0) \\ 0 \quad(\Delta E<0) .\end{cases}$ 


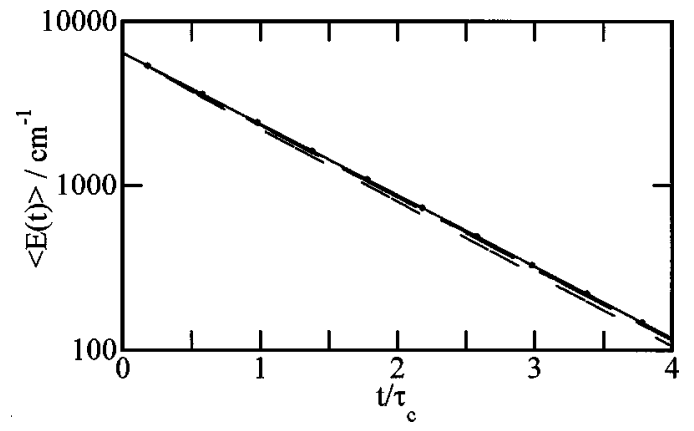

FIG. 11. A Monte Carlo simulation of the apparent relaxation of the vibrational excess energy in the presence of the excited state lifetime that depends on the vibrational excess energy. Solid curve with filled circles stands for the correct time dependence $\left[\langle E(0)\rangle \exp \left(-t / \tau_{c}\right)\right.$, where $\tau_{c}$ means the vibrational cooling time]. Dotted, dashed, dashed-dotted, and long-dashed curves are the results of simulations at $\tau_{c}=100 \mathrm{ps}, 500 \mathrm{ps}, 1 \mathrm{~ns}$, and $2 \mathrm{~ns}$, respectively.

We consider that this exponential distribution suitable for the first approximation. ${ }^{6}$ The averaged energy loss $(\langle\Delta E\rangle(E))$ is proportional to the excess energy $(E)$ in our model as follows:

$$
\langle\Delta E\rangle(E)=\frac{E}{Z \tau_{c}},
$$

where $\tau_{c}$ and $Z$ stand for the cooling time and the collision frequency, respectively. Upward collisions (collisional activation) are not considered in our model for simplicity. In our simulation, a solute molecule is prepared as the excited state with an initial energy, $6430 \mathrm{~cm}^{-1}$. Then collisions with buffer gases are generated randomly with collision frequency $Z$, and the vibrational excess energy $(e(t))$ decreases at each collision according to the probability distribution as Eq. (A1). At the same time, the electronic relaxation from the $S_{2}$ state occurs according to the energy dependent lifetime as Eq. (1) with experimentally determined values of $\tau_{F}^{0}$ and $\alpha$. Therefore, the survival probability $(p(t))$ of the $S_{2}$ state decreases with time. The averaged vibrational excess energy $(\langle E(t)\rangle)$ in our experiment is expressed by $p(t)$ and $e(t)$ as follows:

$$
\langle E(t)\rangle=\frac{\langle p(t) e(t)\rangle}{\langle p(t)\rangle} .
$$

On the other hand, what we should obtain actually is $\langle e(t)\rangle$, which obeys the following time dependence:

$$
\langle e(t)\rangle=e(0) \exp \left(-\frac{t}{\tau_{c}}\right) .
$$

These two functions do not agree with each other exactly, so long as the electronic relaxation and the vibrational excess energy are correlated.

We have calculated $\langle E(t)\rangle$ with different values of $\tau_{c}$, and the results are compared with Eq. (A4) numerically. The value of $Z \tau_{c}$ is fixed to 100 , which is a typical value of our experiment (Table I). The averages are taken for 1000 trajectories. The results of the simulations are shown in Fig. 11. The values of $\langle E(t)\rangle$ agree well with Eq. (A4). In particular, both functions are almost indistinguishable when the cooling time $\left(\tau_{c}\right)$ is smaller than $1 \mathrm{~ns}$. It indicates that the approximation of Eq. (7) works quantitatively in most of our experimental conditions.

${ }^{1}$ (a) D. C. Tardy and B. S. Rabinovitch, Chem. Rev. 77, 369 (1977), and references therein; (b) I. Oref and D. C. Tardy, ibid. 90, 1407 (1990), and references therein.

${ }^{2}$ (a) D. W. Oxtoby, Annu. Rev. Phys. Chem. 32, 77 (1981), and references therein; (b) D. W. Oxtoby, Adv. Chem. Phys. 47, 487 (1981), and references therein.

${ }^{3}$ T. Elsaesser and W. Kaiser, Annu. Rev. Phys. Chem. 42, 83 (1991), and references therein.

${ }^{4}$ (a) H. Hippler, J. Troe, and H. J. Wendelken, J. Chem. Phys. 78, 6718 (1983); (b) H. Hippler, L. Lindemann, and J. Troe, ibid. 83, 3906 (1985); (c) M. Damm, F. Deckert, H. Hippler, and J. Troe, J. Phys. Chem. 95, 2005 (1991).

${ }^{5}$ D. Schwarzer, J. Troe, M. Votsmeier, and M. Zerezke, J. Chem. Phys. 105, 3121 (1996).

${ }^{6}$ U. Hold, T. Lenzer, K. Luther, K. Reihs, and A. Symonds, Ber. Bunsenges. Phys. Chem. 101, 552 (1997).

${ }^{7}$ (a) D. R. McDowell, F. Wu, and R. B. Weiseman, J. Chem. Phys. 108, 9404 (1998); (b) F. Wu and R. B. Weiseman, ibid. 110, 5047 (1999).

${ }^{8}$ (a) M. Fraelich, M. S. Eliot, and A. S. Mullin, J. Phys. Chem. A 102, 9761 (1998); (b) M. S. Eliot, M. Fraelich, R. L. Sansom, and A. S. Mullin, J. Chem. Phys. 111, 3517 (1999).

${ }^{9}$ D. C. Tardy, J. Chem. Phys. 99, 963 (1993).

${ }^{10}$ J. R. Gascooke, Z. T. Alwahabi, K. D. King, and W. D. Lawrance, J. Phys. Chem. A 102, 8505 (1998).

${ }^{11}$ W. D. Lawrance and A. E. W. Knight, J. Chem. Phys. 79, 6030 (1983).

${ }^{12}$ S. H. Kable, J. W. Thoman, Jr., and A. E. W. Knight, J. Chem. Phys. 88, 4748 (1988).

${ }^{13}$ R. N. Schwartz and K. F. Herzfeld, J. Chem. Phys. 22, 767 (1954).

${ }^{14}$ M. S. Eliot, M. C. Wall, A. S. Lemoff, and A. S. Mullin, J. Chem. Phys. 110, 5578 (1998).

${ }^{15}$ G. V. Hartland, D. Qin, and H.-L. Dai, J. Chem. Phys. 102, 8677 (1995).

${ }^{16}$ B. M. Toselli and J. R. Barker, J. Chem. Phys. 97, 1809 (1992).

${ }^{17}$ A. Chimbayo, B. M. Toselli, and J. R. Barker, J. Chem. Phys. 108, 2383 (1998).

${ }^{18}$ M. Châtelet, J. Kieffer, and B. Oksengorn, Chem. Phys. 79, 413 (1983).

${ }^{19}$ M. E. Paige and C. B. Harris, Chem. Phys. 149, 37 (1990).

${ }^{20}$ K. E. Schultz, D. J. Russel, and C. B. Harris, J. Chem. Phys. 97, 5431 (1992).

${ }^{21}$ (a) D. Schwarzer, J. Troe, and M. Zerezke, J. Chem. Phys. 107, 8380 (1997); (b) D. Schwarzer, J. Troe, M. Votsmeier, and M. Zerezke, Ber. Bunsenges. Phys. Chem. 101, 595 (1997).

${ }^{22}$ C. Kittel, J. Chem. Phys. 14, 614 (1946).

${ }^{23}$ (a) T. A. Litovitz, J. Chem. Phys. 26, 469 (1957); (b) W. Madigosky and T. A. Litovitz, ibid. 34, 489 (1961).

${ }^{24}$ D. W. Oxtoby, Mol. Phys. 34, 987 (1977).

${ }^{25}$ R. Biswas, S. Bhattacharyya, and B. Bagchi, J. Chem. Phys. 108, 4963 (1998).

${ }^{26}$ (a) S. A. Adelman, R. Muralidhar, and R. H. Stote, J. Chem. Phys. 95, 2738 (1991); (b) S. A. Adelman, R. H. Stote, and R. Muralidhar, ibid. 99, 1320 (1993).

${ }^{27}$ G. Goodyear and R. M. Stratt, J. Chem. Phys. 107, 3098 (1997).

${ }^{28}$ J. Benzler, S. Linkersdörfer, and K. Luther, J. Chem. Phys. 106, 4992 (1997).

${ }^{29}$ G. Goodyear and S. C. Tucker, J. Chem. Phys. 110, 3643 (1999).

${ }^{30}$ R. Zwanzig, J. Chem. Phys. 34, 1931 (1961).

${ }^{31}$ H. K. Shin and J. Keiser, Chem. Phys. Lett. 27, 611 (1974).

${ }^{32}$ B. J. Cherayil and M. D. Fayer, J. Chem. Phys. 107, 7642 (1997).

${ }^{33}$ U. Sukowski, A. Seilmeier, T. Elsaesser, and S. F. Fischer, J. Chem. Phys. 93, 4094 (1990).

${ }^{34}$ K. Iwata and H. Hamaguchi, J. Phys. Chem. A 101, 632 (1997)

35 (a) M. Terazima, J. Chem. Phys. 189, 793 (1996); (b) M. Terazima, Chem. Phys. Lett. 305, 189 (1999); (c) T. Okazaki, N. Hirota, and M. Terazima, J. Chem. Phys. 110, 11399 (1999).

${ }^{36}$ (a) C. Heidelbach, J. Schroeder, D. Schwarzer, and V. S. Vikhrenko, Chem. Phys. Lett. 291, 333 (1998); (b) C. Heidelbach, I. I. Fedchenia, D. Schwarzer, and J. Schroeder, J. Chem. Phys. 108, 10152 (1998); (c) C. Heidelbach, V. S. Vikhrenko, D. Schwarzer, and J. Schroeder, ibid. 110, 5286 (1999); (d) C. Heidelbach, V. S. Vikhrenko, D. Schwarzer, I. I. Fedchenia, and J. Schroeder, ibid. 111, 8022 (1999). 
${ }^{37}$ (a) M. Shiga and S. Okazaki, J. Chem. Phys. 109, 3542 (1998); (b) M. Shiga and S. Okazaki, ibid. 111, 5390 (1999).

${ }^{38}$ A. Morita and S. Kato, J. Chem. Phys. 109, 5511 (1998).

${ }^{39}$ B. M. Ladanyi and R. M. Stratt, J. Chem. Phys. 111, 2008 (1999).

${ }^{40}$ T. Yamaguchi, Y. Kimura, and N. Hirota, J. Chem. Phys. (in press).

${ }^{41}$ Y. Kimura, T. Yamaguchi, and N. Hirota, Chem. Phys. Lett. 303, 223 (1999).

${ }^{42}$ D. J. Muller, W. D. Lawrance, and A. E. W. Knight, J. Phys. Chem. 87, 4952 (1983).

${ }^{43}$ W. Jalenak, R. E. Weston, Jr., and T. J. Sears, J. Chem. Phys. 89, 2015 (1988).

${ }^{44}$ M. Fujii, T. Ebata, N. Mikami, and M. Ito, Chem. Phys. 77, 191 (1983).

${ }^{45}$ Y. Hirata and E. C. Lim, J. Chem. Phys. 69, 3292 (1978).

${ }^{46}$ W. D. Lawrance and A. E. W. Knight, J. Phys. Chem. 94, 1249 (1990).

${ }^{47}$ G. Orlandi and F. Zerbetto, Chem. Phys. 113, 167 (1987).

${ }^{48}$ K. Ohta, T. J. Kang, K. Tominaga, and K. Yoshihara, Chem. Phys. 242, 103 (1999).

${ }^{49}$ N. Sarkar, S. Takeuchi, and T. Tahara, J. Phys. Chem. A 103, 4808 (1999).

${ }^{50}$ K.-J. Choi, B. P. Boczar, and M. R. Topp, Chem. Phys. 57, 415 (1981).

${ }^{51}$ J. B. Hopkins and P. M. Rentzepis, Chem. Phys. Lett. 117, 414 (1985).

${ }^{52}$ Y. Kimura and N. Hirota, J. Chem. Phys. 111, 5474 (1999).

${ }^{53}$ M. D. Dawson, T. F. Boggess, and A. L. Smirl, Opt. Lett. 12, 254 (1987).

${ }^{54} \mathrm{We}$ set the excitation polarization to the magic angle with respect to the line between the sample and the slit of the spectrograph, so that the effect of rotational diffusion diminishes. Although the rotational relaxation does not affect the fluorescence bandshape, it alters the time profile of the fluorescence intensity at the earlier stage. Since we determined the response function from the rise of the total fluorescence intensity, the rotational relaxation can alter the apparent response function. The time profile of the fluorescence intensity can also affect the apparent cooling rates through $f(t)$ in Eq. (7). In order to test these effects, we also performed the experiment with vertical and horizontal excitations in liquids. We found that the apparent cooling rate changes with excitation polarization by about $\pm 1 \mathrm{ps}$ in methanol, ethylene glycol, and water, whereas no meaningful changes are found in cyclohexane and acetonitrile. The apparent rates become faster in the case of vertical excitation, whereas they become slower in the case of horizontal excitation. The direction of these changes agrees with the theoretical prediction.

${ }^{55}$ C. J. Sansonetti, M. L. Salit, and J. Reader, Appl. Opt. 35, 74 (1996).

${ }^{56}$ W. H. Melhuish, Appl. Opt. 14, 26 (1975).

${ }^{57}$ T. Yamaguchi, Y. Kimura, and N. Hirota, J. Phys. Chem. A 101, 9050 (1997).

${ }^{58}$ Helium: R. D. McCarty, J. Phys. Chem. Ref. Data 2, 923 (1973). Argon: C. H. Twu, L. L. Lee, and K. E. Starling, Fluid Phase Equilibria 4, 35 (1980). Xenon: V. A. Rabinovich, A. A. Vasserman, V. I. Nedstup, and L.
S. Veksler, Thermophysical Properties of Neon, Argon, Krypton, and Xenon (Hemisphere, Washington, DC, 1988). Nitrogen: T. H. Chung, M. M. Khan, L. L. Lee, and K. E. Starling, Fluid Phase Equilibria 17, 351 (1984). Ethane: B. A. Younglove and J. F. Ely, J. Phys. Chem. Ref. Data 16, 577 (1987). Carbon Dioxide: F. H. Huang, M. H. Li, K. E. Starling, and F. T. H. Chun, J. Chem. Eng. Jpn. 18, 490 (1985). Water: Kagaku Binran, 4th ed. (Chemical Society of Japan, Tokyo, 1993).

${ }^{59}$ In the previous letter (Ref. 41) we used the second order polynomial instead. The fitting is not so good at the lower excess energy in the case of the second order polynomial, and this discrepancy leads to the overestimate of the relaxation rate in the high-density fluid.

${ }^{60} \mathrm{We}$ consider that this assumption is not valid, and the invalidity appears as the nonproportionality of the relaxation rates with the solvent density. The nonproportionality has also been found for the vibrational energy relaxation rates in the ground state (Ref. 5) and we consider that it is due to the nonlinear dependence of the collision frequency on the solvent density. Since the equations of state evidently deviates from that of ideal gas in this density region, it is quite natural that the collision frequency is not proportional to the solvent density, either. However, we consider that this error in the estimation of $Z_{\mathrm{LJ}}$ is small enough to discuss the relative rates among different quenchers and the difference between two electronic states.

${ }^{61}$ T. Itoh, J. Phys. Chem. A 103, 2247 (1999).

${ }^{62}$ H.-K. Nienhuys, S. Woutersen, R. A. van Santen, and H. J. Bakker, J. Chem. Phys. 111, 1494 (1999).

${ }^{63}$ Q. Zhong, Z. Wang, Y. Sun, Q. Zhu, and F. Kong, Chem. Phys. Lett. 248, 277 (1996).

${ }^{64}$ (a) T. Nakabayashi, H. Okamoto, and M. Tasumi, J. Phys. Chem. A 101, 7189 (1997); (b) T. Nakabayashi, H. Okamoto, and M. Tasumi, ibid. 102, 9686 (1998).

${ }^{65}$ Y. Mizutani, Y. Uesugi, and T. Kitagawa, J. Chem. Phys. 111, 8950 (1999).

${ }^{66}$ D. R. Demmer, J. W. Hager, G. W. Leach, and S. C. Wallace, Chem. Phys. Lett. 136, 329 (1987).

${ }^{67}$ E. W.-G. Diau, S. De Feyter, and A. H. Zewail, J. Chem. Phys. 110, 9785 (1999).

${ }^{68}$ M. L. Horng, J. A. Gardecki, A. Papazyan, and M. Maroncelli, J. Phys. Chem. 99, 17311 (1995).

${ }^{69}$ J. E. Haebig, J. Phys. Chem. 71, 4203 (1967).

${ }^{70}$ However, it should be noted that Rector et al. reported that the population decay time $\left(T_{1}\right)$ of overtone is smaller than the half of $T_{1}$ of fundamental at the low temperature [K. D. Rector, A. S. Kwok, C. Ferrante, A. Tokmakoff, C. W. Rella, and M. D. Feyer, J. Chem. Phys. 106, 10027 (1997)], although $T_{1}$ in their experiment may not correspond to the intramolecular energy transfer. 